\title{
An anesthetic experience with cesarean section in a patient with vasovagal syncope
} -A case report-

\author{
Seung Yong Park, and Seong Su Kim
}

Department of Anesthesiology and Pain Medicine, Gangneung Asan Hospital, Ulsan University College of Medicine, Gangneung, Korea

Epidural anesthesia was performed for a cesarean section in a patient with vasovagal syncope. A 26-year-old woman at 41 weeks of gestation and with a known history of vasovagal syncope was scheduled to undergo an emergency cesarean section. She received epidural anesthesia for the cesarean delivery and showed signs and symptoms of vasovagal syncope, which were treated successfully with fluids and the administration of a vasoconstrictor. This case highlights the need for anesthesiologists to understand vasovagal syncope precisely because severe hypotension in a patient under anesthesia for a cesarean section is dangerous to both the mother and baby. (Korean J Anesthesiol 2010; 59: 130-134)

Key Words: Cesarean section, Epidural anesthesia, Vasovagal syncope.

Vasovagal syncope (neurocardiogenic syncope) is defined as a sudden and transient loss of consciousness. It is caused by an abnormal or exaggerated autonomic response to a range of stimuli of which the most common are an erect posture and emotional upsets. It manifests clinically as hypotension associated with paradoxical bradycardia, heart block or sinus arrest $[1,2]$. The onset of syncope is relatively rapid and the recovery is spontaneous, complete and usually prompt. Therefore, this clinical disorder is often not taken seriously by either the patients or physicians. Although the patient recovers consciousness rapidly and spontaneously, repeated episodes can cause a wide variety of medical problems ranging from mild hypotension to serious cardiac asystole. In addition, the patients can suffer from trauma or motor vehicle accidents during sudden unexpected syncopal episodes.

Many procedures and conditions in the anesthetic rooms can also cause vasovagal episodes (e.g., venipuncture, placement of epidural catheter, anxiety). These episodes may also occur during regional anesthesia, in association with hemorrhage or supine inferior vena cava compression during pregnancy, which can be additive when combined [3]. Syncopal attack in pregnant women can cause severe problems to both the mother

Received: August 14, 2009. Revised: 1st, September 8, 2009; 2nd, September 23, 2009. Accepted: October $20,2009$.

Corresponding author: Seong Su Kim, M.D., Department of Anesthesiology and Pain Medicine, Gangneung Asan Hospital, Ulsan University College of Medicine, 415, Bangdong-ri, Sacheon-myeon, Gangneung 210-711, Korea. Tel: 82-33-610-3402, Fax: 82-33-641-8180, E-mail: sskim@gnah.co.kr (c) This is an open-access article distributed under the terms of the Creative Commons Attribution Non-Commercial License (http:// creativecommons.org/licenses/by-nc/3.0/), which permits unrestricted non-commercial use, distribution, and reproduction in any medium, provided the original work is properly cited. 
and baby, including unconsciousness, pulmonary aspiration, apnea, even cardiac arrest to mother and fetal hypoxia, acidosis, and neurological injury to the baby. During regional anesthesia for a cesarean section, the combination of vasovagal activity and sympathetic block can worsen the problem. Therefore, when a pregnant woman has a history of syncope and shows vasovagal attacks, the vasovagal activity should be suppressed readily and the procedure completed as quickly as possible. There are several reports on the management of patients with vasovagal syncope during pregnancy and labor in the international literature but none in Korea. We encountered a patient with vasovagal syncope scheduled to undergo epidural anesthesia for a cesarean section. We report this case with a review of the relevant literature.

\section{Case Report}

A 26-year-old woman at 41 weeks of gestation with a known history of vasovagal syncope was scheduled to undergo an emergency cesarean section due to fetal distress. She weighed $92 \mathrm{~kg}$ and was $165 \mathrm{~cm}$ tall. Two years earlier, she visited the hospital with complaints of frequent abdominal discomfort, cold extremities, cold sweating, and a brief loss of consciousness. Such symptoms began $4-5$ years earlier. They lasted for 5-10 minutes and resolved spontaneously. She experienced episodes of syncope that was induced by many conditions. She was diagnosed with vasovagal syncope by a cardiologist and neurologist through a careful history and physical examination, echocardiography, treadmill test, Holter monitoring and positive tilt table test, and a $\beta$-blocker was prescribed (atenolol $25 \mathrm{mg} / \mathrm{d}$ ). She stopped medication herself after a few days. The other pre-anesthetic assessment was unremarkable, with no medical problems except dizziness and healthy pregnancy. Initially, she planned a normal vaginal delivery under epidural analgesia. With the patient in the left lateral decubitus position and using an aseptic technique, a lumbar epidural catheter was inserted at the L3-4 interspace with rapid fluid administration and tested with $1 \%$ lidocaine $3 \mathrm{ml}$. She did not show any changes in vital signs, and was observed without a further epidural injection until a cervical dilatation of $5-6 \mathrm{~cm}$.

She complained dizziness twice, which was resolved with bed rest and atenolol medication. During non-invasive fetal monitoring, the fetus showed variable heart rate deceleration and an emergency cesarean section was decided. No premedication was prescribed. She had a heart rate of 60-115 beats/min and an arterial pressure of 90-110/60-90 $\mathrm{mmHg}$ at the delivery unit. When the patient arrived in the anesthetic room, monitoring of the ECG, oxygen saturation and arterial pressure was started, showing a heart rate of 75 beats/min and an arterial pressure 120/80 mmHg. Because epidural catheter was already inserted, it was decided to proceed with the operation under epidural anesthesia. After placing the patient in the supine position with a left lateral tilt, a 20-gauge catheter was inserted at left radial artery after modified Allen's test for direct arterial pressure monitoring. A 16-gauge peripheral line was inserted and Hartmann's solution $600 \mathrm{ml}$ was administered. Oxygen was administered via a face mask. After a negative aspiration test, $2 \%$ lidocaine $20 \mathrm{ml}$ plus fentanyl $100 \mu \mathrm{g}$ was injected through the epidural catheter. Ten minutes after the epidural injection, she had a heart rate and arterial pressure of 74 beats/min and $89 / 54 \mathrm{mmHg}$, respectively. Accordingly, ephedrine $5 \mathrm{mg}$ was injected with rapid fluid administration. The anesthetic level was T5 to light touch with an alcohol sponge at 15 minutes after the epidural injection, and a cesarean section proceeded rapidly without complications. A male infant was delivered with a 1 and 5 minutes Apgar score of 7 and 9 . The baby was ventilated manually with a bag and a mask due to cyanosis, and transferred to the neonatal ICU. He weighed 3,510 $\mathrm{g}$ and showed mild cardiomegaly and a ductal aneurysm on the echocardiographic examination. Over a 2-day admission, the baby showed a normal muscle tone and respiratory pattern. After delivery 10 units oxytocin was infused with fluids. The decrease in heart rate and blood pressure to 50 beats/min and 76/40 $\mathrm{mmHg}$, respectively, was treated with rapid fluids administration, intravenous glycopyrrolate $0.2 \mathrm{mg}$ and ephedrine $5 \mathrm{mg}$ intravenously three times. She complained of dizziness that disappeared after normalizing the blood pressure. The total operation time was 80 minutes and 1,900 ml of Hartmann's solution was infused. Epidural PCA with $0.15 \%$ ropivacaine plus fentanyl was connected for postoperative pain control. The patient did not show any changes in their vital signs or syncopal attack at recovery room over a 60 minutes observation period and was transferred to the general ward.

\section{Discussion}

Vasovagal syncope has a familial tendency but is not believed to be genetically inherited. It can be initiated by external stimuli or by stimulation of the Bezold-Jarisch reflex. The characteristics of vasovagal syncope are severe hypotension with paradoxical bradycardia after a transient period of sympathetic excitation, which ultimately leads to syncope. Stimulation of the cardiac C-fibers is implicated in vasovagal syncope. In the presence of hypovolemia and other conditions leading to a reduction in preload, the sympathetic tone is increased due to stimulation of the ventricular mechanoreceptors, resulting in hypercontractility of the volume empty left ventricle. The subsequent stimulation of the cardiac C-fibers results in a combination of parasympathetic overactivity (bradycardia) and 
decreased sympathetic tone (hypotension) with the ensuing syncope. Hypotension is likely the primary event, even in patients with bradycardia or asystole, whereas vagally-mediated bradycardia may play only a secondary role in the pathogenesis of vasovagal syncope $[1,4]$. Therefore, preventing and correcting the hypotension is of most importance. A number of other neurohumoral agonists, such as endogenous opioid peptides, vasopressin, serotonin, endothelin, and nitric oxide, also have been proposed to play a role in the pathogenesis of vasovagal syncope [5]. The patient generally shows palpitation before the syncopal attack or fainting. The same condition could be produced during the preoperative tilt test with isoproterenol, which is consistent with the characteristics of vasovagal syncope.

A preoperative determination of the autonomic nervous system regulation may provide an opportunity to detect patients at risk of significant hemodynamic compromise and guide prophylactic therapy with either volume prehydration or vasopressor infusion. In addition, it may decrease the risk of hypotension as well as the adverse effects of these measures [6]. The table tilt test is a generally useful and non-invasive method for measuring the activity of the autonomic nervous system during an analysis of heart rate variability (HRV). The variability is affected by a range of factors, such as the volume status, respiration, intrathoracic pressure, and baroreceptor reflexes [7]. Hypotension accompanying spinal anesthesia can occur due to hypovolemia or an autonomic nervous system instability, which is a condition reflected by decreased HRV [8]. HRV measurements may contain important prognostic information about the individual reactions. Frölich and Caton [9] reported that the heart rate and blood pressure in a prehydrated pregnant patient increased in response to an orthostatic challenge. A higher baseline heart rate prior to hydration in pregnant women scheduled to undergo an elective cesarean section using spinal anesthesia was associated with more hypotension and less hemodynamic stability. The bispectral index (BIS) can also be used to monitor a syncopal attack. Win et al. [10] reported a decreased BIS to be an indicator of syncope before hypotension and bradycardia in patients with needle phobia.

The higher risk of maternal complications associated with general anesthesia compared to regional anesthesia has led to the increased use of subarachnoid block and epidural anesthesia for both elective and emergency cesarean deliveries [6]. Although pregnant women are known to have increased sympathetic activity, hypotension is a common adverse effect of a central neuraxial block and it is mainly the result of decreased systemic vascular resistance after the blockade of preganglionic sympathetic fibers. However, no prophylactic strategy to prevent hypotension, intravenous fluids or vasopressors, has proven to be entirely satisfactory and applicable to all patients.
Therefore, many pregnant patients develop hypotension during a central neuraxial block despite the prophylactic measures, whereas other patients are at risk of side effects from excessive prehydration or vasopressor infusion [6]. Severe hypotension of the mother impairs placental perfusion, which can lead to fetal hypoxia, acidosis and neurological injury. There is a report that maternal hypotension due to vasovagal attack can cause abruptio placenta [11]. Therefore, maternal hypotension should be treated rapidly for both maternal and fetal wellbeing.

A loss of consciousness in patients with vasovagal syncope may be preceded by a brief prodrome of nausea, diaphoresis, light-headedness, blurred vision, headache, palpitations, paresthesia and pallor, which normally occur in the upright position and resolve immediately when the patient assumes the supine position. The differential diagnosis includes carotid sinus hypersensitivity and orthostatic hypotension. In this case, the patient complained of such symptoms but it appeared more frequently during voiding and defecation, or when she had a gastric upset rather than when she was in the erect position. Therefore, orthostatic hypotension could be ruled out. The head up tilt table test (HUT) also revealed symptoms during an isoproterenol infusion, but not for the simple table tilt condition. HUT can also be used to assess the effectiveness of a contemplated treatment. There are several reports on the repeated tilt testing, and continued standing training can restore the normal orthostatic tolerance. The patient can learn to recognize the onset of syncopal symptoms and avoid a loss of consciousness through education during the diagnostic and therapeutic procedure.

There is some debate regarding how syncopal attacks in pregnant women can be better managed. There is no evidence that operative delivery is safer, and it was decided to aim for a spontaneous vaginal delivery under epidural analgesia. Epidural analgesia can reduce the risk of syncopal attacks through the relief of labor pain. Closed monitoring and maintaining the blood pressure during epidural analgesia are essential. Before introducing the epidural catheter, the patient was given enough information about her condition and our delivery plan, and was reassured. This may help reduce the patient's anxiety and risk of syncopal attacks. Continuous ECG, oxygen saturation, direct blood pressure, and fetal monitoring may be helpful during epidural analgesia. A sympathetic block after an epidural injection will cause vasodilatation. Accordingly, a large-bore intravenous catheter should be inserted and fluids administered to maintain the hydration status and avoid hypovolemia. Some recommend that a cesarean section can be delayed while anticholinergic and sedative premedications are administered. However, although anticholinergic premedication may prevent vagally mediated bradycardia it does not prevent hypotension, which is the likely primary event. While there is no rationale 
to suspect that a larger fluid preload might have prevented a vagal reaction, it might have reduced the hemodynamic consequences. Subsequently, a slow onset regional block using the epidural alone might have been more appropriate than spinal anesthesia. It was reported that general anesthesia can also be conducted safely in patients without cardiac disease to facilitate rapid delivery in the event of hemodynamic collapse.

Several anesthetic measures were planned based on several reports. There are no clear guidelines for anesthetizing patients with vasovagal syncope, particularly in an obstetric setting. There are several reports related to anesthetic management of obstetric patients. Watkins et al. [12] reported a patient who developed vasovagal syncope during epidural and spinal anesthesia for a cesarean section. Saqr and Kumar [13] reported the successful management of two patients with vasovagal syncope who received epidural anesthesia for a cesarean section and epidural analgesia for a vaginal delivery. They reported that spinal anesthesia is clearly not a good option because sudden vasodilation would precipitate an episode of syncope. However, general anesthesia would be safe if conducted in a cardio-stable manner. Anxiolysis, avoiding the supine position and compression stockings are non-pharmacological methods. Many reports have stated that pretreatment with glycopyrrolate and light sedation are not enough to suppress the excessive vagal tone caused by the patient's anxiety.

An epidural test injection after inserting the epidural catheter was satisfactory and there were no signs of a rapid motor block or a high sensory block to suggest subarachnoid catheter placement. The sensory block was not increased over the T5 level. The patient was kept in the supine left lateral tilt position. When the patient complained of dizziness and became pale, she denied any symptoms, such as perioral numbness, tingling, or a metallic taste that indicated a vascular injection. The combination of vasovagal activity and sympathetic block, with or without aortocaval compression could be a contributing factor. The vasovagal activity was mostly doubled, except for the absence of severe bradycardia. It is believed that it was masked transiently by ephedrine and may be due to the injection of glycopyrrolate, continuous ECG and direct blood pressure monitoring. This means that most of the vasovagal activity can be suppressed readily with ephedrine and atropine if close hemodynamic monitoring is used. Oxytocin must be used with caution as it can cause vasodilation and a sudden decrease in blood pressure. Intensive monitoring should be continued and the patients nursed in a close observation unit for a further 2448 hours. Drug prefilled syringes (e.g., atropine, ephedrine) and equipment for resuscitation should be kept at hand. Adequate postoperative pain control is also important for preventing vasovagal attack.

Vasovagal syncope has a favorable long-term prognosis when diagnosed and treated appropriately. A standard treatment for the prevention of vasovagal syncope has not been established. The best option is to complete the procedure as quickly as possible. There are number of therapeutic options available, which are non-pharmacological or pharmacological. Educating the patient to avoid predisposing conditions, such as dehydration, stress, excessive alcohol consumption, an extreme warm environment and tight clothing, remains the mainstay of the treatment. Reassuring the patient about the benign nature of the condition along with anxiety management will be helpful. Fluid and sodium loading along with the use of compression stockings to reduce venous pooling can help avoid syncopal attacks. Drug therapy may include $\beta$-blockers, $\alpha$-agonists, selective serotonin reuptake inhibitors, hydrocortisone, midodrine, theophylline, disopyramide, scopolamine and hyoscyamine [1]. Because of their relatively low risk, $\beta$-blockers are the most widely used drug for the prevention of vasovagal syncope. However, its long term use is controversial. Against all appearances, the choice of $\beta$-blockers for treatment appears logical because both spontaneous and tilt-induced syncope are preceded by elevated levels of catecholamines. Although permanent pacemakers are effective in patients whose syncope is refractory and has a significant cardio-inhibitory component, the cardiac pacing is not effective in most patients [4].

In conclusion, pain and anxiety can cause a vasovagal attack during the induction of regional anesthesia as with general anesthesia. Vasovagal episodes superimposed on regional anesthesia are a significant hazard, particularly in pregnant women. Attention to a history of syncopal episodes may help identify patients at risk of recurrent vasovagal attacks. Anesthetists should try to prevent the condition with proper communication before the procedure. Adequate positioning and reassurance to relieve anxiety may be a useful adjunct to a successful regional block. Careful hemodynamic monitoring and constant vigilance for sudden bradycardia are essential during regional anesthesia. Early aggressive management of any vasovagal episode is recommended and rapid delivery of the infant may be necessary.

\section{References}

1. Chen-Scarabelli C, Scarabelli TM. Neurocardiogenic syncope. BMJ 2004; 329: 336-41.

2. Fitzpatrick AP, Cooper P. Diagnosis and management of patients with blackouts. Heart 2006; 92: 559-68.

3. Kinsella SM, Tuckey JP. Perioperative bradycardia and asystole: relationship to vasovagal syncope and the Bezold-Jarisch reflex. Br J Anaesth 2001; 86: 859-68.

4. Sra JS, Jazayeri MR, Avitall B, Dhala A, Deshpande S, Blanck Z, et al. Comparison of cardiac pacing with drug therapy in the treatment of neurocardiogenic (vasovagal) syncope with bradycardia or asystole. 
N Engl J Med 1993; 328: 1085-90.

5. Robertson RM, Medina E, Shah N, Furlan R, Mosqueda-Garcia R. Neurally mediated syncope: pathophysiology and implications for treatment. Am J Med Sci 1999; 317: 102-9.

6. Hanss R, Bein B, Ledowski T, Lehmkuhl M, Ohnesorge H, Scherkl W, et al. Heart rate variability predicts severe hypotension after spinal anesthesia for elective cesarean delivery. Anesthesiology 2005; 102: 1086-93.

7. Hanss R, Bein B, Francksen H, Scherkl W, Bauer M, Doerges V, et al. Heart rate variability-guided prophylactic treatment of severe hypotension after subarachnoid block for elective cesarean delivery. Anesthesiology 2006; 104: 635-43.

8. Chamchad D, Arkoosh VA, Horrow JC, Buxbaum JL, Izrailtyan I, Nakhamchik L, et al. Using heart rate variability to stratify risk of obstetric patients undergoing spinal anesthesia. Anesth Analg 2004; 99: 1818-21.
9. Frölich MA, Caton D. Baseline heart rate may predict hypotension after spinal anesthesia in prehydrated obstetrical patients. Can J Anaesth 2002; 49: 185-9.

10. Win NN, Kohase H, Miyamoto T, Umino M. Decreased bispectral index as an indicator of syncope before hypotension and bradycardia in two patients with needle phobia. Br J Anaesth 2003; 91: 749-52.

11. Weksler N, Rozenstweig V, Shapira AR. Severe vasovagal attack: an unusual cause of abruptio placentae. Arch Gynecol Obstet 2004; 270: 299-301.

12. Watkins EJ, Dresner M, Calow CE. Severe vasovagal attack during regional anaesthesia for Caesarean section. Br J Anaesth 2000; 84 118-20.

13. Saqr L, Kumar MM. Neurocardiogenic syncope in the obstetric patient. Anaesthesia 2007; 62: 79-84. 